\title{
Human agency beliefs influence behaviour during virtual social interactions
}

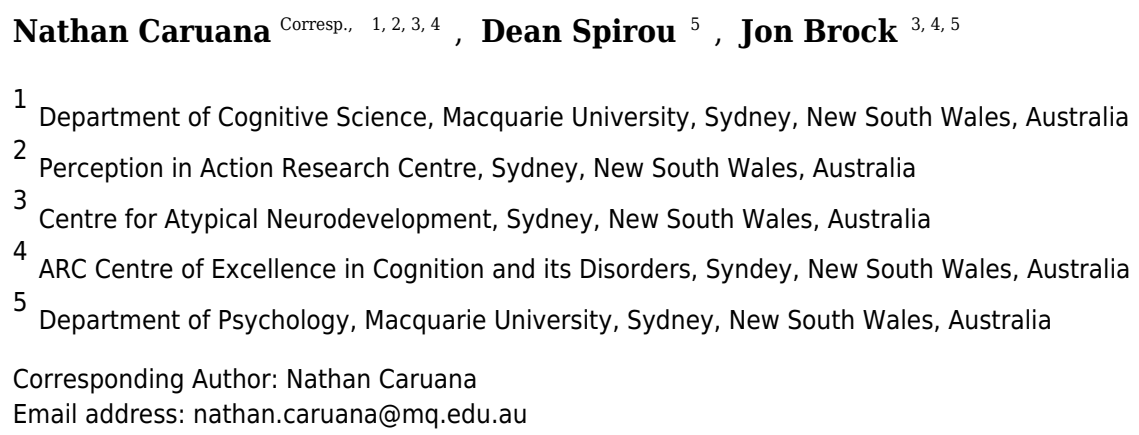

In recent years, with the emergence of relatively inexpensive and accessible virtual reality technologies, it is now possible to deliver compelling and realistic simulations of human-tohuman interaction. Neuroimaging studies have shown that, when participants believe they are interacting via a virtual interface with another human agent, they show different patterns of brain activity compared to when they know that their virtual partner is computer-controlled. The suggestion is that users adopt an "intentional stance" by attributing mental states to their virtual partner. However, it remains unclear how beliefs in the agency of a virtual partner influence participants' behaviour and subjective experience of the interaction. We investigated this issue in the context of a cooperative "joint attention" game in which participants interacted via an eye tracker with a virtual onscreen partner, directing each other's eye gaze to different screen locations. Half of the participants were correctly informed that their partner was controlled by a computer algorithm ("Computer" condition). The other half were misled into believing that the virtual character was controlled by a second participant in another room ("Human" condition). Those in the "Human" condition were slower to make eye contact with their partner and more likely to try and guide their partner before they had established mutual eye contact than participants in the "Computer" condition. They also responded more rapidly when their partner was guiding them, although the same effect was also found for a control condition in which they responded to an arrow cue. Results confirm the influence of human agency beliefs on behaviour in this virtual social interaction context. They further suggest that researchers and developers attempting to simulate social interactions should consider the impact of agency beliefs on user experience in other social contexts, and their effect on the achievement of the application's goals. 
1

2

3

4

5

6

7

8

9

10

11

12

13

Corresponding author: Dr. Nathan Caruana

$14 * \mathrm{NC}$ and DS contributed equally to this work

15

16

\section{Human Agency Beliefs Influence Behaviour during Virtual Social Interactions}

\author{
Nathan Caruana 1234
}

Dean Spirou*5

Jon Brock ${ }^{2} 45$

${ }_{2} \mathrm{ARC}$ Centre of Excellence in Cognition and its Disorders, MACQUARIE UNIVERSITY, Sydney

${ }_{3}$ Perception in Action Research Centre, MACQUARIE UNIVERSITY, Sydney

${ }_{4}$ Centre for Atypical Neurodevelopment, MACQUARIE UNIVERSITY, Sydney

${ }_{5}$ Department of Psychology, MACQUARIE UNIVERSITY, Sydney 


\section{Abstract}

18 In recent years, with the emergence of relatively inexpensive and accessible virtual reality

19 technologies, it is now possible to deliver compelling and realistic simulations of human-to-

20 human interaction. Neuroimaging studies have shown that, when participants believe they are

21 interacting via a virtual interface with another human agent, they show different patterns of brain

22 activity compared to when they know that their virtual partner is computer-controlled. The

23 suggestion is that users adopt an "intentional stance" by attributing mental states to their virtual

24 partner. However, it remains unclear how beliefs in the agency of a virtual partner influence

25 participants' behaviour and subjective experience of the interaction. We investigated this issue in

26 the context of a cooperative "joint attention" game in which participants interacted via an eye

27 tracker with a virtual onscreen partner, directing each other's eye gaze to different screen

28 locations. Half of the participants were correctly informed that their partner was controlled by a

29 computer algorithm ("Computer" condition). The other half were misled into believing that the

30 virtual character was controlled by a second participant in another room ("Human" condition).

31 Those in the "Human" condition were slower to make eye contact with their partner and more

32 likely to try and guide their partner before they had established mutual eye contact than

33 participants in the "Computer" condition. They also responded more rapidly when their partner

34 was guiding them, although the same effect was also found for a control condition in which they

35 responded to an arrow cue. Results confirm the influence of human agency beliefs on behaviour

36 in this virtual social interaction context. They further suggest that researchers and developers

37 attempting to simulate social interactions should consider the impact of agency beliefs on user

38 experience in other social contexts, and their effect on the achievement of the application's goals. 


\section{Human Agency Beliefs Influence Behaviour during Virtual Social Interactions}

The development in recent years of relatively inexpensive and accessible virtual reality

43

44

45

technology now makes it possible to deliver compelling and realistic simulations of human-to-

human interaction (Georgescu, Kuzmanovic, Roth, Bente, \& Vogeley, 2014; Schroeder, 2002).

Potential applications of virtual social interaction are only starting to be explored, but already include gaming, market research, basic and clinical scientific research, military simulation training, long distance health care delivery and education (Lee \& Stewart, 2016). Some of these applications involve the co-presence of two or more human-controlled avatars within the same virtual environment. However, when the interaction is to be delivered to a large number of users (e.g., for standardised education and training delivery) or when tight control of the interaction is required (e.g., in social cognition and neuroscience research), it may be possible and desirable for individual humans to interact with a virtual character whose behaviour is entirely controlled by a computer algorithm (Caruana, McArthur, Woolgar \& Brock, 2017a; Georgescu et al., 2014). In such contexts, an important question is the extent to which the user experience is affected by their knowledge that the social interaction is artificial. In other words, does the user interact differently with a virtual social partner if they know that the partner is a computercontrolled agent rather than a human-controlled avatar?

Central to this question is the observation that humans negotiate everyday social interactions by mentalising - interpreting the behaviour of social partners in terms of mental states such as beliefs, desires, and goals - and then using those inferred mental states to predict future behaviour and adapt their responsive behaviour accordingly (Premack \& Woodruff, 1978). To give a concrete example, suppose you are walking towards someone on a crowded footpath, 
63 and your eyes meet. At once, you are mutually aware of each other and can make a joint effort to

64 avoid bumping into one another. However, if you see that the other person is looking at the

65 displays in the shop windows as they walk down the path, you will predict that they will continue

66 to walk towards you unaware, and must change your own trajectory to avoid a collision. If we

67 believe that a virtual character is controlled by another human then we are likely to engage in

68 these same mentalising processes, adopting what philosophers refer to as an "intentional stance",

69 because we see the agent's behaviour as a product of an intentional and intelligent "mind"

70 (Wykowska, Wiese, Prosser, \& Muller, 2014). The question is, what happens when we know or

71 believe that the virtual character is artificial? Does our interpretation of its behaviour - and

72 therefore our response - change? Or does a sufficiently realistic virtual partner elicit the adoption

73 of an intentional stance even when we consciously know that our partner lacks human agency

74 and, therefore, mental states?

75 In the current study, we addressed these questions in the context of a gaze-based "joint

76 attention" interaction - in which participants interacted with a virtual partner to reach a common

77 focus of attention. In a typical joint attention episode, one person initiates joint attention (IJA) by

78 directing their partner's attention to an object or location in space (Bruinsma, Koegel, \& Koegel,

79 2004). The second person responds to the joint attention bid (RJA), and, finally, the first person

80 monitors the behaviour of the second to determine whether joint attention has been achieved

81 (Bruinsma, Koegel, \& Koegel, 2004). Joint attention is reciprocal, dynamic, and intentional

82 (Schilbach et al., 2013). It also requires individuals to represent the mental states of others (e.g.,

83 What is my partner looking at? Are they attempting to communicate with me? etc.). Thus, joint

84 attention provides a useful model of social interaction for investigating the effects of agency

85 beliefs during virtual interactions. 

one's own eye movements will be seen by another person in a two-way interaction) can influence communicative eye movement behaviour (Gobel, Kim \& Richardson, 2015). Until now, however, the gaze processing literature has largely been restricted to the investigation of gaze perception in non-interactive contexts (see Schilbach et al., 2013 for a review). This highlights the need to investigate the influence of social context on interactive gaze behaviour where eye gaze is simultaneously used as a cue to understand others (perception) as well as a communication mechanism (signaling; Gobel et al., 2015). By using interactive tasks which measure joint attention behaviour, we are able to investigate the influence that human agency beliefs have on the way we perceive and respond to gaze (RJA) and use our own gaze to communicate (IJA). (see Caruana, et al., 2017a) in which participants' eye-movements are tracked as they interact with an animated virtual character, whose own eye-movements are responsive to those of the participant. In some studies, participants have been told that the "avatar" is controlled by a second participant whose eye movements are also being recorded (e.g., Schilbach et al., 2010). In other studies, participants know that their partner is computer-controlled (e.g., Oberwelland et al., 2016). Recently, two studies have directly investigated whether these different approaches, and the adoption of human agency beliefs, influence brain activity during joint attention

107 experiences. In a study by Pfeiffer and colleagues (2014) participants initiated joint attention bids 108 towards a target, and their virtual partner responded by either looking towards or away from the 
109 target. For each block of trials, participants were required to indicate whether they believed their

110 virtual partner was being controlled by a human or computer. Although, in reality, the virtual

111 character was always computer-controlled, blocks of trials in which participants believed they

112 were interacting with another human were associated with increased activation of the ventral

113 striatum - a brain region associated with reward processing. However, in this study, agency

114 beliefs were confounded with task success (i.e., achieving joint attention), as participants in the

115 naïve condition were more likely to say that the avatar was human-controlled on blocks when he

116 was more responsive. Thus, striatal activity may simply reflect task success irrespective of

117 agency beliefs.

118 In a second study measuring event-related potentials (ERPs), we employed a similar task

119 and a between-subjects design, informing half of the participants that the virtual character was

120 human-controlled and half that he was computer-controlled (Caruana, de Lissa \& McArthur,

121 2017). We found that the N170 - an early occipitotemporal brain response to visual information

122 - was larger in response to gaze shifts in the group who believed the virtual character was

123 human-controlled (see Wykowska et al., 2014 for similar findings). We also found that the P350

124 - a later response measured over centro-parietal sites - was sensitive to joint attention success

125 only in the group who believed that the virtual character was human-controlled. As with the

126 study by Pfeiffer and colleagues, the differential brain response suggests that participants process

127 the outcome of a joint attention episode differently depending on their beliefs in the agency of

128 their partner. However, it is important to note that, in both cases, the effect is driven by the

129 behaviour of the virtual partner - whether he is programmed to respond correctly or not on each

130 trial. These studies do not address the impact of agency beliefs on participants' own behaviour

131 during the interaction; that is, how they respond to, and initiate joint attention bids. 
In the current study, therefore, we investigated whether human agency beliefs have a

133 direct influence on joint attention behaviour. As in the studies reviewed above, participants

134 interacted with a virtual partner in a cooperative joint attention game. Half of the participants

135 believed that their partner was controlled by another human (Human condition). The remainder

136 were correctly informed that their partner was computer-controlled (Computer condition). Their

137 task was to catch a burglar located in one of six houses placed around the edge of the screen (see

138 Figure 1). At the start of each trial, the participant and their partner searched their allotted houses

139 and whomever found the burglar was then required to look back at the burglar to signal its

140 location. The burglar was caught when both players were looking at the correct location. Unlike

141 previous joint attention studies investigating the influence of human agency beliefs, this task

142 created a context in which sometimes the participant found the burglar and had to "Initiate" joint

143 attention, and other trials where they did not find the burglar, and had to "Respond" to their

144 partner instead. In addition to this "Social" task, participants also completed a non-social

145 "Control” task in which the virtual character's eyes remained closed and participants completed

146 the same sequence of eye-movements in response to geometric shape cues (circles and arrows).

147 We have used this task in previous studies but without the agency manipulation. In other

148 words, all participants believed that they were interacting with a real person (Caruana, Brock \&

149 Woolgar, 2015; Caruana, Steiglitz Ham et al., 2017; Caruana, McArthur, Woolgar and Brock,

150 2017b). These studies have produced a number of consistent findings which motivated our

151 predictions in the current study.

152 First, on responding (RJA) trials, participants are slower to respond to their partner's eye

153 gaze cue than for the equivalent arrow cue in the control (RJAc) condition. Importantly, this

154 effect is reduced when the search phase is removed from the task so that the virtual partner only 
155 makes a single eye-movement on each trial (Caruana et al., 2017b). This suggests that an

156 important part of the joint attention task is determining whether a shift in eye gaze is intended to

157 be communicative or not. If participants know that their partner is not human and, therefore, has

158 no mental states or intentions, they may not evaluate the communicative intent of their partner's

159 behaviour in the same way. We therefore predicted that this effect would be reduced in the

160 Computer condition compared to the Human condition..

161 Second, on "Initiate" trials, participants discover the burglar and are then required to look

162 back towards the avatar. We find they are slower to do this in the Social (IJA) condition than the

163 Nonsocial (IJAc) control condition. They are then required to either wait for eye contact from

164 their partner (IJA) or wait for the central fixation point to turn green (IJAc) before saccading

165 back to the burglar location. We have found that participants make more premature saccades

166 (i.e., failing to wait for the respective cue before looking back at the burglar) in the IJA condition

167 compared to IJAc. Again, these findings can be interpreted in terms of the inferred mental states

168 of the virtual partner. When participants think their partner is human, they assume that he will

169 intuitively know that they are looking at a location to initiate joint attention, even when eye

170 contact is not first established to signal their own communicative intent. In the Control condition,

171 they know they are interacting with the computer and so approach the task quite differently,

172 making the same robotic pattern of eye movements on each trial. Our prediction, therefore, is

173 that both of these effects will be reduced when participants know that their virtual partner is

174 computer-controlled. That is, they will approach the interaction with the virtual partner in a

175 similar fashion to their "interaction" with the symbols on the screen. If these predictions were

176 confirmed, they would provide the first direct evidence that beliefs about the human agency of

177 virtual characters can influence user behaviour during virtual interactions. 
179

180

181

182

183

184

185

186

187

188

189

190

191

192

193

194

195

196

197

198

199

200

\section{Ethical statement}

This study was approved by the Macquarie University Human Research Ethics

Committee (Approval reference: 5201200021). All participants provided verbal and written consent before participating in the study.

\section{Participants}

Participants were first year undergraduate students at Macquarie University who received course credit for their involvement. They were alternately allocated to either the "human" or "computer" group in the order of participation. At the end of the experiment, two participants in the human group indicated that they did not believe that a human was controlling the virtual character. These two participants were excluded and replaced by the next participant in the testing schedule. The final sample included 48 participants, 24 in each group. The two groups were similar in terms of sex ratio (19 females in human group, 17 in the computer group) and age (Human: $M=19.33, S D=0.52$; Computer: $M=19.51, S D=0.35$ ). All participants had normal vision and reported having no psychiatric diagnoses or history of neurological impairment or injury. All participants were right-handed, as confirmed using the Edinburgh Handedness Inventory (Oldfield, 1971).

\section{Joint Attention Task}

At the beginning of each session all participants completed the Oldfield Handedness Inventory. During this time, the experimenter (DS) told participants in the human condition that he was going to "check-up" on a colleague who would be assisting with the study, briefly left the room, entered the adjacent room for a minute, and then returned. The experimenter then read the same set of scripted instructions to participants in both groups using graphical cue cards (see 
201 Supplementary Material 1, cards 1, 7-12). Participants in the human group were then told that the

202 experimenter's colleague, Alan, who was in the adjacent eye-tracking laboratory, would be

203 controlling the avatar that they would be completing the task with. Participants were also told

204 that their eye movements would be displayed on a virtual character that Alan could see on his

205 screen. This deception was supported by additional instruction cards (Supplementary Material 1, 206 cards 2-6) which explained how the interactive interface worked and illustrated Alan's view of

207 the stimulus. Participants in the computer group were simply told (truthfully) that the avatar was 208 controlled by a computer program.

209 The joint attention task was programmed using Experiment Builder 1.10.165 software

210 (SR Research, 2004). It was identical for both groups (human and computer) and was also

211 identical to that used in our previous studies (Caruana et al., 2015; Caruana, Steiglitz Ham et al.,

212 2017; Caruana et al., 2017b). Full details of the gaze-contingent algorithm can be found in

213 Caruana et al. (2015).

214 The display comprised an anthropomorphic virtual character in the center of the screen 215 subtending 6.5 degrees of visual angle (see Supplementary Material 2 for images of all avatar 216 stimuli), with two horizontal rows of three houses, each subtending four degrees of visual angle, 217 positioned above and below the virtual character (see Figure 1). At the beginning of each trial, 218 participants were required to search the houses with a blue door by fixating them in turn, 219 whereupon the doors open to reveal either an empty house or the burglar. The location of the 220 blue doors (i.e., top versus bottom row of houses) changed from the first to the second block, and 221 block order was counterbalanced across participants within each group. On some trials, one or 222 two houses were already open to vary the participants' search behaviour across trials.

223 Participants could search these houses in any order they chose. 


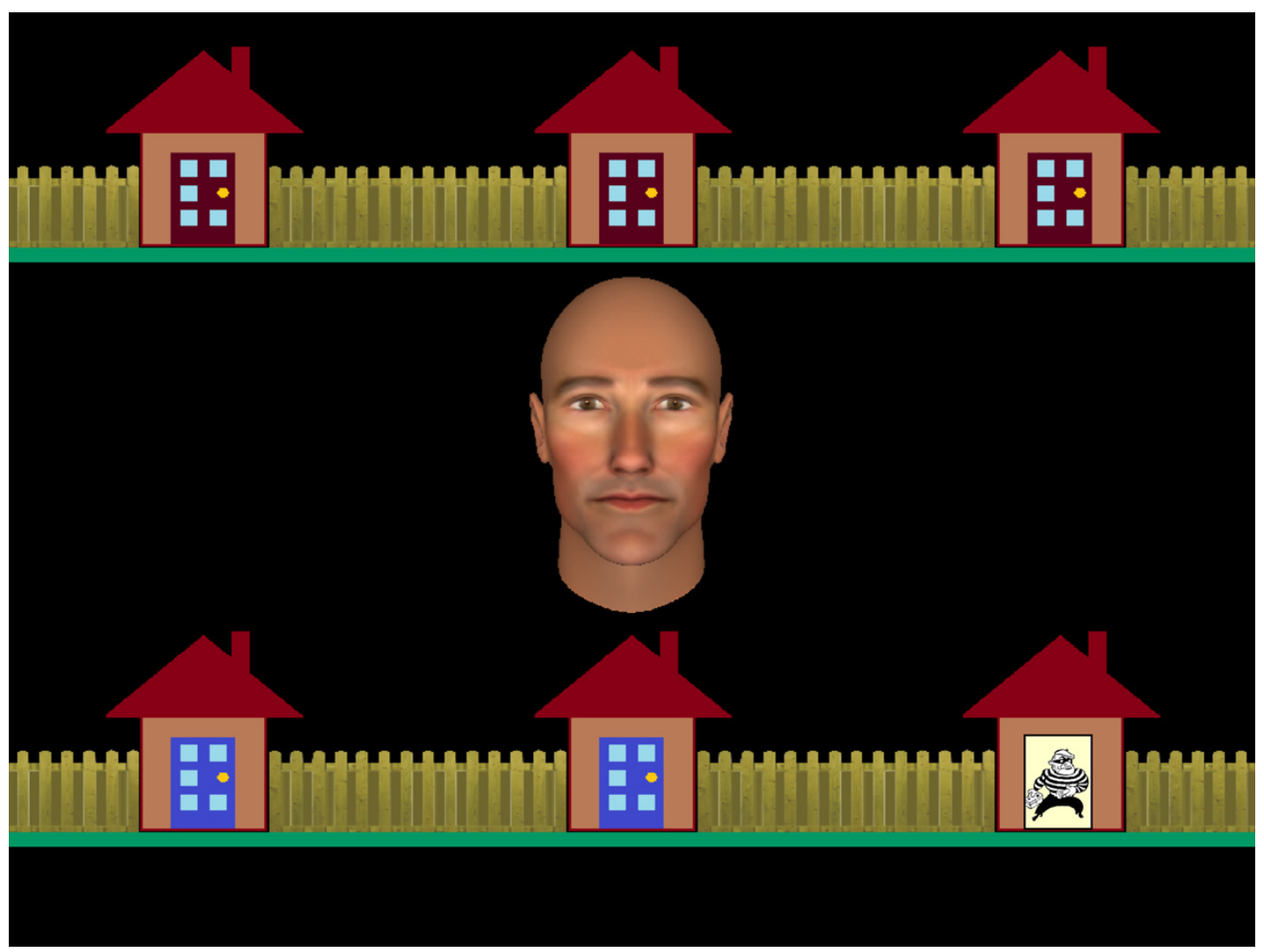

Figure 1. A screenshot of the interactive task stimuli. The row of houses with blue doors are to be searched by the

226 participant to find the burglar. The houses with red doors are to be searched by the virtual partner depicted by the

227 virtual character in the centre of the screen.

Social conditions (RJA and IJA).

Once the participant completed their search (either by finding the burglar or by

231 discovering that all the blue houses were empty), they were required to look back at their partner

232 to establish eye contact. The virtual character was programmed to search the red-doored houses

233 in a random order until the participant had looked back at them and then to make 0-2 additional

234 gaze shifts before establishing eye contact. The onset latency of each gaze shift varied between

$235500-1000 \mathrm{~ms}$. This meant that the delay between fixating the avatar's face and the establishment

236 of eye contact varied between 500-3000ms. 
238 blue-doored houses empty, indicating that the burglar was in one of the red-doored houses. Once

239 eye contact had been established, the virtual character would look towards the red door

240 concealing the burglar. If the participant responded by looking at that door, it would open to

241 reveal the burglar behind bars to indicate that he had been captured.

242 Initiating joint attention (IJA). In IJA trials, the participant would find the burglar in one

243 of the blue-doored houses. Following eye contact, the participant was required to conduct an

244 "initiating saccade" to the location of the burglar by fixating back on the house that contained the

245 burglar. At this point, the virtual character would follow the participant's gaze. If this was the

246 correct location, then the burglar again reappeared behind bars. Importantly, the virtual character

247 did not respond if the participant made their initiating saccade prior to the establishment of eye

248 contact (this was classified as a premature saccade). However, the trial could still be completed if

249 the participant looked back at their partner, established eye contact, and made a second initiating 250 saccade back to the burglar.

251 Feedback. On correct trials, participants were informed that they had successfully caught

252 the burglar if the burglar appeared behind bars. On incorrect trials, participants were presented

253 with the burglar in red at the correct location to indicate that they were unsuccessful in catching

254 the burglar. An incorrect trial could be the result of a 'location error' or a 'timeout error'. A

255 location error occurred when participants fixated the wrong location when responding to or

256 initiating a joint attention bid. A timeout error occurred when participants failed to respond to or

257 initiate a joint attention bid within three seconds of being guided on RJA trials, and establishing

258 eye contact on IJA trials respectively. Finally, a Search Error occurred, and participants were

259 presented with a "Failed Search" error message if they spent more than three seconds fixating 
260 away from their designated houses before completing their search for the burglar. If this

261 occurred, the trial was terminated and removed from all analyses.

262

Non-social conditions (RJAc and IJAc). To control for the non-social task requirements

263 in both the RJA and IJA task conditions (e.g., task complexity, attention and action inhibition),

264 two non-social conditions were included. In these conditions, participants completed the same

265 task without any social interaction. The virtual character stimulus remained on the screen,

266 however the eyes were closed for the duration of the trial. A grey fixation point was placed in the

267 center of the animated face, which participants were required to fixate once completing their

268 search for the burglar. This turned green after 500-3000ms (analogous to establishing eye

269 contact). On RJAc trials this was followed by the presentation of a green arrow, which indicated

270 the burglar's location (analogous to the virtual partner's guiding eye gaze), which participants

271 were to follow. On IJAc trials, participants were required to fixate back on the burglar's location

272 to catch the burglar once the fixation point turned green.

273 Procedure. Participants completed two blocks, each comprising 108 trials. This included

27427 trials per condition (i.e., IJA, RJA, IJAc RJAc). Within each block, Social (IJA, RJA) and

275 Control (IJAc, RJAc) trials, were completed in clusters of six trials each. Whether each block

276 began with a Social or Control cluster of trials was counterbalanced across subjects and matched

277 between groups. The start of a Social cluster was cued with text reading "Together" and the start

278 of a Control cluster was cued with text reading "Alone". These cues were presented in the centre

279 of the computer screen for 1000 milliseconds each time.

280 Eye-tracking. An EyeLink 1000 Remote Eye-Tracking System (SR Research Ltd.,

281 Ontario, Canada) was used to track the participants' right eye movements with a sampling rate of 
$282500 \mathrm{~Hz}$, and a chin rest to stabilise head movements and standardise viewing distance. A 9-point

283 eye-tracking calibration and validation was conducted at the beginning of each block.

284 Subjective experience ratings and debrief. Following the completion of the joint

285 attention task, participants completed a post-experimental interview where they were asked to

286 rate how difficult, natural, intuitive and pleasant they found the Social and Control tasks on a 10-

287 point Likert scale $(1=$ Not at all, $10=$ Extremely $)$. Participants also rated how co-operative their

288 partner was on Social trials, and how "human-like" the virtual character felt generally, as well as

289 how human-like he appeared and behaved, using the same 10-point scale. Participants were also

290 asked whether they preferred completing the task alone (Control trials) or together with their

291 partner (Social trials), and rated the strength of this preference on a 10-point scale $(1=$

292 completely prefer together, $10=$ completely prefer alone). Participants in both the Human and

293 Computer group were asked the same questions. The questions were designed to gauge the

294 extent to which participants anthropomorphised the virtual character during their interaction with

295 him, and to provide participants in the Human group with the opportunity to disclose whether

296 they had any doubts that they were truly interacting with another human being.

297 At the end of the session, participants in the Human group were debriefed about the true

298 nature of the interaction. At this point they were asked whether they believed they were

299 interacting with another person named Alan. Participants also rated how convinced had been on

300 the same 10-point scale described above.

301 Analysis

302 We used DataViewer software (SR Research Ltd., Ontario, Canada) to export Interest

303 Area and Trial reports. All subsequent analyses were performed in R using a custom script. Raw

304 data and R code can be downloaded from the Open Science Framework: 
305 https://osf.io/yqb7g/?view only=3a14468af22b4465920962ee289ea742. R Markdown can also

306 be viewed here: http://rpubs.com/JonBrock/Belief.

Following our previous studies (Caruana et al. 2015; Caruana, Stieglitz Ham et al., 2017;

308

309

310

312

313

Caruana et al., 2017b), we excluded all trials in which a recalibration was required or an error occurred during the search phase. We then measured the following indices of performance:

Accuracy. Proportion of trials on which the participant successfully caught the burglar. Saccadic reaction time (Respond trials). Mean duration between the presentation of the gaze (RJA) or arrow (RJAc) cue and the onset of the participant's responding saccade to the burglar location. Trials with incorrect responses or reaction times below $150 \mathrm{~ms}$ were excluded. The trial timed out at $3000 \mathrm{~ms}$ (and was coded as an error).

Target dwell time (Initiate trials). Mean duration between the burglar being revealed and the participant looking back towards Alan (IJA) or the fixation point (IJAc). Trials with dwell times below $150 \mathrm{~ms}$ or above $3000 \mathrm{~ms}$ were excluded.

Premature initiating saccades (Initiate trials). Proportion of trials in which a saccade was made from the avatar (IJA) or fixation point (IJAc) to the location of the burglar, prior to the establishment of eye contact (IJA) or the grey fixation point turning green (IJAc).

\section{Statistical Analysis}

Statistical analyses were conducted using the ez package (version 4.4-0) in R. We conducted mixed-ANOVA with condition (i.e., Social versus Control) as a within-subjects factor and group (i.e., Human versus Computer) as a between-subjects factor. We have reported generalised eta squared $\left(\eta_{G}^{2}\right)$ as a measure of effect size. Interactions were followed up with ttests. For the subjective ratings, we used non-parametric Mann-Whitney U tests to investigate the 
327 effect of group for each rating. For Accuracy analyses, we also examined the additional factor of

328 subject role (i.e., Responding vs Initiating).

329

Results

330

331

332

334

335

337

338

339

340
Figure 2 summarizes accuracy by group and condition. Participants made significantly more errors on Social trials (RJA, IJA) than Control trials (RJAc, IJAc) overall, (main effect of condition, $\left.\mathrm{F}(1,46)=43.86, \mathrm{p}<.001, \eta_{\mathrm{p}}{ }^{2}=0.14\right)$, and more errors on Responding trials (RJA, RJAc) than Initiating trials (IJA, IJAc) overall (main effect of social role, F $(1,46)=95.87, \mathrm{p}<$ $\left..001, \eta_{\mathrm{p}}^{2}=0.14\right)$. There was also a significant group*condition*subject role interaction $(\mathrm{F}(1,46)$ $\left.=0.36 .69, \mathrm{p}<.001, \eta_{\mathrm{p}}{ }^{2}=0.15\right)$ which arose because participants made more errors on RJA trials than any other condition. There were no significant main effects of group, nor any significant interactions involving group (all $p \mathrm{~s}>0.08$, see http://rpubs.com/JonBrock/Belief for full analyses).

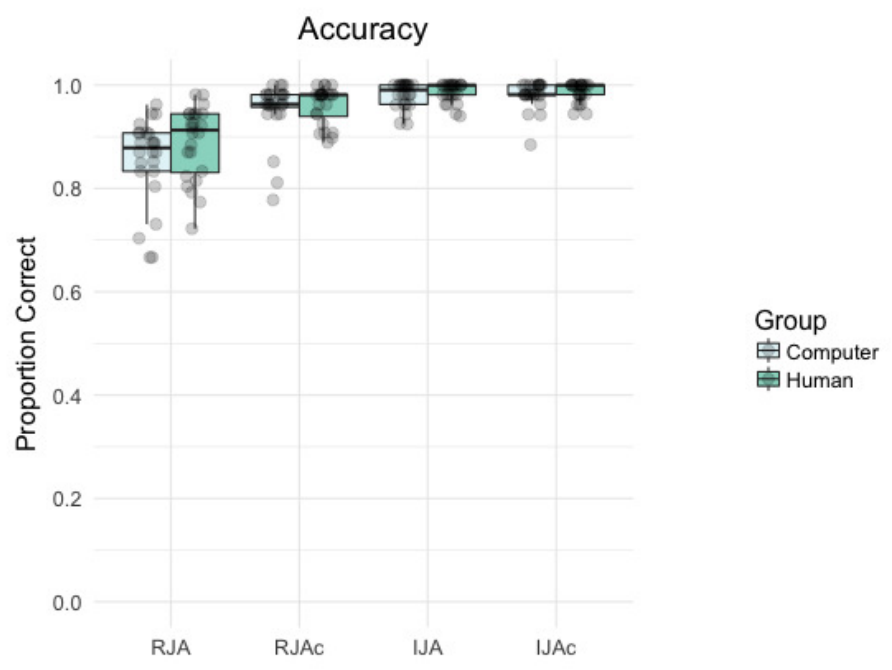

342 Figure 2. Boxplot with individual data points depicting the proportion of correct trials by group (i.e., Computer, 
343 Human) and condition (i.e., RJA, RJAc, IJA, IJAc). In all figures, whiskers extend (as in a conventional Tukey

344 boxplot) to the furthest data points that are within 1.5 times the length of the box from the end of the box.

345

346 Responding to Joint Attention

Saccadic reaction time. Figure $3 \mathrm{~A}$ summarises saccadic reaction time data by group and condition. Participants' saccadic reaction times were significantly slower on RJA trials relative to RJAc trials (main effect of condition, $F(1,46)=264.63, p<.001, \eta_{G}^{2}=0.66$ ). Overall,

351 (main effect of group, $F(1,46)=5.71, p=.021, \eta_{G}^{2}=0.08$ ). However, there was no significant

352 group* condition interaction $\left(F(1,46)=2.34, p=.133, \eta_{G}^{2}=.02\right)$.
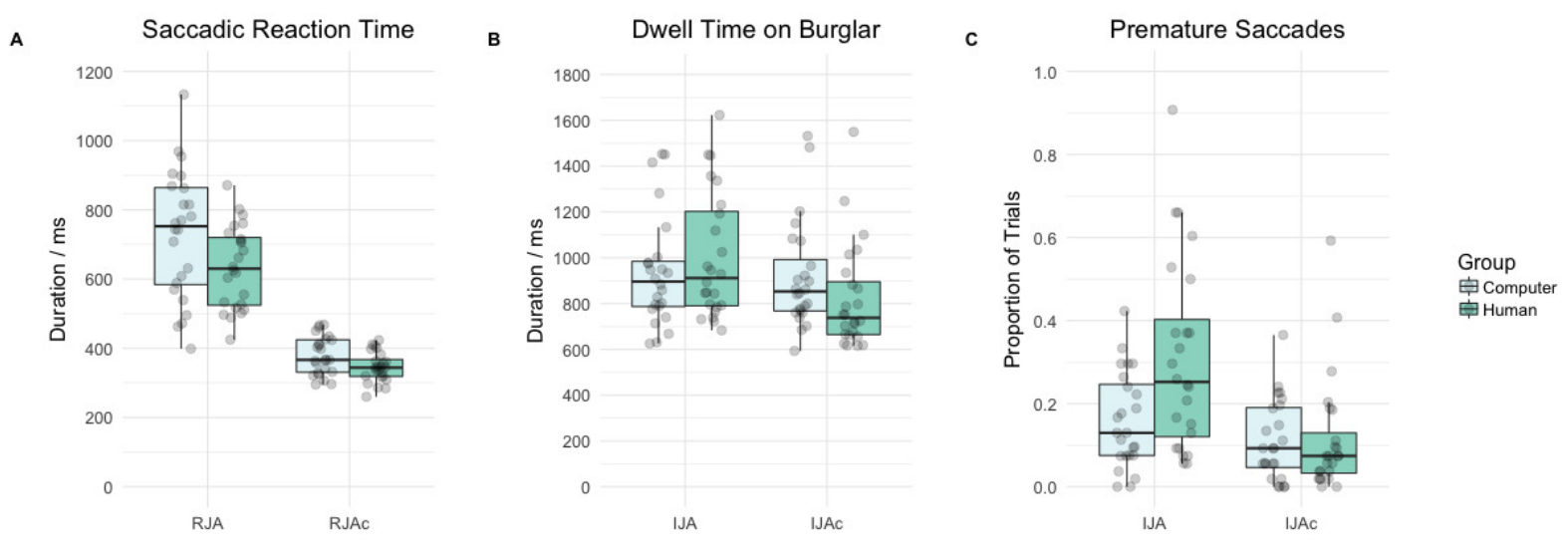

353

Figure 3. Tukey boxplot with individual data points depicting (A) saccadic reaction times in milliseconds (B) dwell time on the burglar in milliseconds and (C) the proportion of trials participants made a premature saccade by group (i.e., Computer, Human) and condition (i.e., RJA versus RJAc for A, or IJA versus IJAc for B and C).

357

\section{Initiating Joint attention}


condition. There was no significant main effect of group $\left(F(1,46)=0.06, p=.816, \eta_{G}^{2}=0.00\right)$.

361 However, as anticipated, participants had significantly longer dwell times for the burglar on IJA

362 trials compared to IJAc trials (main effect of condition, $F(1,46)=24.36, p<.001, \eta_{G}^{2}=0.04$ ).

363 More importantly, in line with our hypotheses, there was also a significant group*condition

364 interaction $\left(F(1,46)=14.72, p<.001, \eta_{G}^{2}=0.03\right)$. Follow-up t-tests revealed that participants

365 had significantly longer dwell times on the burglar on IJA trials compared to IJAc trials, in the

366 Human group $(t(23)=5.58, p<.001)$ but not in the Computer group $(t(23)=0.89, p=.383)$.

368 trials in which participants made a premature initiating saccade, by group and condition. Again,

369 there was no significant main effect of group $\left(F(1,46)=3.78, p=.058, \eta_{G}^{2}=0.06\right)$. However, as

370 predicted, participants made significantly more premature initiating saccades on IJA trials than

371 on IJAc trials (main effect of condition, $F(1,46)=38.50, p<.001, \eta_{G}^{2}=0.14$ ). Of greater

372 interest, and again aligning with our hypotheses, we found evidence of a significant

373 group*condition interaction $\left(F(1,46)=13.79, p<.001, \eta_{G}^{2}=0.06\right)$. Follow-up t-tests revealed

374 that participants made significantly more premature initiating saccades on IJA trials compared to 375 IJAc trials in the Human group $(t(23)=6.15, p<.001)$ and the Computer group $(t(23)=2.11, p$ $376=.046$,

\section{Subjective Task Ratings}

Figure 4 provides a summary of the subjective task ratings involving the social condition.

379 Participants in the Human group rated their partner as being significantly more cooperative 380 compared to participants in the Computer group $W=193.0, p=.039$. They also found the task 
381 more pleasant, $W=188.5, p=.038$, but less intuitive, $W=384.5, p=.045$. There were no

382 significant differences between groups in any other ratings.

383 Belief of the agency of the virtual character. In the Human group, all participants other

384 than two excluded participants (see Participants section) reported that they were convinced they

385 were interacting with another human being through the virtual interface. 

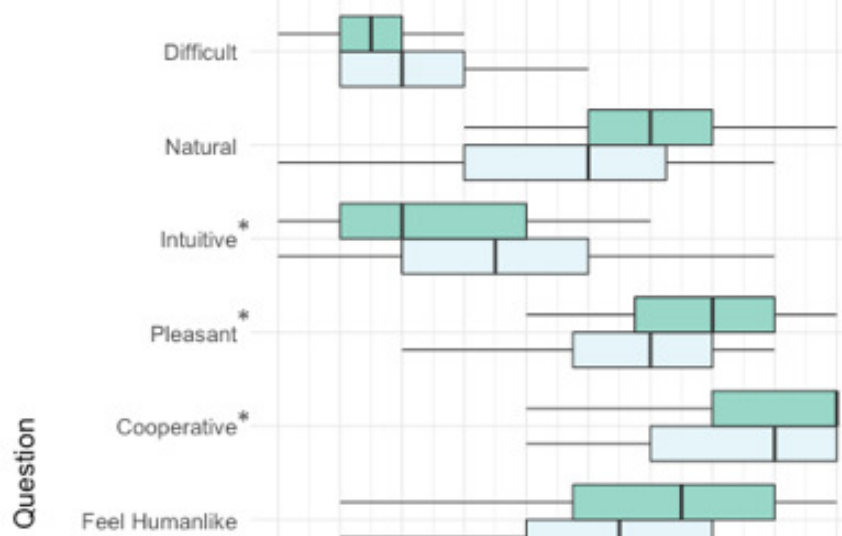

Feel Humanlike

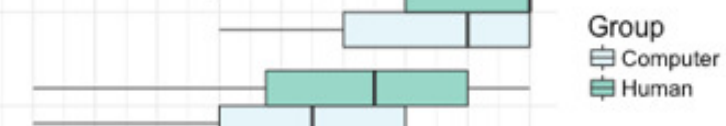

Appear Humanlike
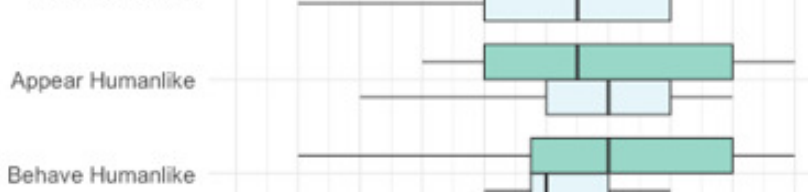

官Computer

Behave Humanike

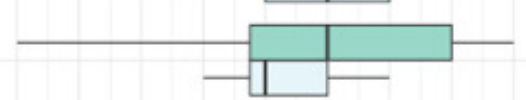

Prefer Alone

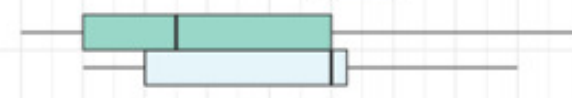

Prefer Virtual

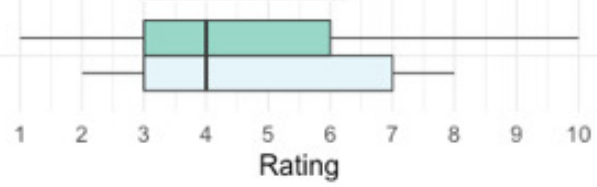

Figure 4. Tukey boxplots depicting responses to subjective ratings questions: (1) Difficult, How difficult did you

find the interactive task? (2) Natural, How natural did the interaction feel? (3) Intuitive, How intuitive was the interactive task? (4) Pleasant, How pleasant was the interaction? (5) Cooperative, How cooperative did you think your partner was? (6) Feel Humanlike, How human-like did the avatar feel? (7) Appear Humanlike, How human-

392 like was the avatar's appearance? (8) Behave Humanlike, How human-like was the avatar's behaviour? (9) Prefer

393 Alone, Which task did you prefer most? The interactive or the solo task? (10) Prefer Virtual, Would you prefer to 394 play this game face-to-face or using virtual reality? 


\section{Discussion}

397

The current study investigated the effect of human agency beliefs on behaviour during

398 virtual joint attention interactions. Although overall task accuracy was equivalent in our two

399 groups, participants who believed that their virtual partner was controlled by a real person

400 showed markedly different patterns of eye-movements and response times compared to

401 participants who knew that their partner was computer-controlled. As discussed below, these

402 findings indicate that human agency beliefs affect expectations of a virtual partner's behaviour,

403 responsiveness and flexibility, as well as perhaps the human's own social motivation.

\section{Responding to Joint Attention}

405

The results observed in the Human group replicated the findings of our previous studies

406

407

408

409

410

411

412

413

414

415

416

417

in which all participants have believed their virtual partner to be human-controlled (Caruana et

al. 2015; Caruana, Stieglitz Ham et al., 2017; Caruana et al., 2017b). In the Respond conditions, participants responded more slowly to the eye gaze cue during the Social condition (RJA) than the arrow cue in the Control condition (RJAc). As noted earlier, this effect is partially attributable to the ambiguity of the eye gaze cues, which occur in the context of multiple noncommunicative eye-movements made by the virtual character. This requires participants to engage in a process that we call "intention monitoring" (see Caruana et al., 2017b) because participants are required to infer the "communicative intent" of their partner's gaze cues. However, contrary to expectation, we did not find a reduction of the effect in the current Computer group. Despite knowing that their partner was not real and therefore had no intentions, participants were still significantly slower to respond to the eye gaze cue than the arrow cue, with the effect being similar in magnitude to the Human condition. 
One explanation for this finding is that the nature of the RJA task uniquely encouraged

419 participants to evaluate the avatar's behaviour in the same way they would an intentional agent.

420 Even though participants in the Computer group did not believe that they were interacting with

421 an intentional agent, they were still required to process the cues that usually signal intentional

422 and communicative social behaviour (i.e., eye contact) in order to selectively respond to the gaze

423 cues that signalled the burglar's location, and disambiguate these joint attention bids from earlier

424 gaze shifts that were part of their partner's search for the burglar. This cue-disambiguation

425 process was absent on the non-social RJAc trials, where participants responded to unambiguous

426 arrow cues, which explains why we see a difference in saccadic response times on RJA and

427 RJAc trials for both groups. The additional task demands placed on participants during RJA

428 trials may also explain why accuracy was significantly lower for these trials compared to RJAc

429 trials across both groups.

$430 \quad$ Although we did not find an interaction between Condition and Group for Responding

431 trials, we did find a main effect of Group with participants in the Human group responding faster

432 on Social and Control trials compared to participants in the Computer group. This may be

433 because participants in the Human group were more motivated than those who believed that they

434 were interacting with a computer. In other words, the perceived presence of a human co-operator

435 produced an 'audience effect' or social pressure which induced faster responses across both

436 social and non-social trials (Park \& Catrambone, 2007). Indeed, this interpretation does align

437 with some of the incidental comments made by some participants in the Human group of this

438 study, and other studies that we have run in the past, in which they would say things like " $I$

439 didn't want to let the other guy down" or "I felt that Alan was better at the task than I was".

440 Initiating Joint Attention. 
Results from the Human group also replicated our previous findings for the Initiating

442 joint attention condition. Firstly, we found no effects of condition (or group) for accuracy on

443 Initiating trials. Consistent with our previous work (cf. Caruana, Steiglitz Ham et al., 2017)

444 ceiling effects were observed across all participants and conditions. This is likely due to the fact

445 that, upon finding the burglar, participants had an unlimited amount of time to complete IJA and

446 IJAc trials. This allowed us to investigate the pattern of eye-movement behaviours reported in

447 our subsequent analyses, but also made it difficult for participants to fail.

448 Compared to the equivalent measures in the Control condition, these participants spent

449 more time looking at the target of joint attention before attempting to establish eye contact. They

450 also made more premature attempts at initiating joint attention before their virtual partner had

451 returned his gaze to establish eye contact (cf. Caruana et al. 2015; Caruana, Stieglitz Ham et al.,

452 2017; Caruana et al., 2017b). As predicted, we found that both effects were reduced in the

453 Computer group. These participants made fewer premature attempts and spent less time looking

454 at the burglar before making eye contact. Importantly (and in contrast to the Responding

455 condition), these effects were specific to the Social condition and could not, therefore, be

456 explained in terms of an audience effect on performance. These findings are consistent with the

457 view that participants in the Human group adopted an "intentional stance" towards the virtual

458 character, and thus, expected their partner to be an intelligent and flexible agent who would

459 follow their gaze cues, whether or not eye contact had been explicitly established.

460 Wykowska and colleagues (2014) have argued that when participants adopt an intentional

461 stance towards an entity, this exerts a "top down" effect on the interaction, guiding the

462 participant's predictions and expectations concerning the entity's behaviour. Thus, when

463 individuals believe they are interacting with a human, they view their partner's behaviours as the 
464 product of an intentional and intelligent mind and engage in the mentalising processes that are

465 normally recruited during human interactions. This in turn reinforces expectations about how the

466 entity should behave. In the current context, this means that participants may have expected their

467 partner to know that a prolonged dwell time on a particular location or rapid looking backward

468 and forward between that location and the face indicated that they had found the burglar, even

469 when eye contact was not explicitly established. In contrast, when interacting with a non-human

470 entity, Wykowska and colleagues suggest that participants adopt a "design stance" in which they

471 view the entity's behaviours as the product of an engineered system. Participants in the

472 Computer group would not, therefore, have formed any expectations of their virtual partner,

473 making them less likely to attempt initiating joint attention before eye contact had been

474 established.

475 This interpretation of the eye-tracking data is also consistent with the subjective task

476 ratings provided by participants at the completion of the experiment. Specifically, those in the

477 Human group rated the Social condition task as being less intuitive than the Computer group.

478 This makes sense, if we interpret the eye-tracking data as indicating a violation of the flexible

479 responsive behaviour that the Human group participants expected from their partner.

480

The current findings compliment the recent neuroimaging studies of virtual joint attention

481 interactions discussed earlier, which indicate that brain responses associated with the successful

482 achievement of joint attention are moderated by beliefs about the human agency of the virtual

483 partner (Pfeiffer et al., 2014; Caruana, de Lissa et al., 2017). Our results are also broadly

484 consistent with earlier studies that investigated the neural correlates of mentalising by

485 manipulating agency beliefs. For example, Gallagher, Jack, Roepstorff and Frith (2002) reported

486 differential brain activity in the anterior paracingulate cortex during a computerised version of 
487 "stone, paper, scissors", depending on whether participants believed they were playing against a

488 human or computer. Similar findings have been reported in other neuroimaging studies involving

489 cooperative games (McCabe, Houser, Ryan, Smith \& Trouard, 2001). However, the current

490 results go further, indicating that human agency beliefs directly influence behaviour - how the

491 participants interact with their virtual partner - and not simply how they evaluate the outcome of

492 that interaction.

493 The results of the study indicate, therefore, that the ecological-validity of a virtual social

494 interaction may depend on whether users believe their virtual partner represents another living

495 human being. The design, development, and implementation of social simulations should

496 therefore include consideration and, if necessary, evaluation of whether human agency beliefs

497 facilitate or mitigate the achievement of the application's goals. The importance of these beliefs

498 is likely to depend on the area of application. For instance, in social cognition and neuroscience

499 research - as we establish directly in this paper - the adoption of human agency beliefs and an

500 intentional stance appears to be an important ingredient when achieving an ecologically-valid

501 measure of social cognition and behaviour (cf. Caruana et al., 2017a). Likewise, it would not be

502 surprising that user behaviour be similarly affected in other social applications of virtual reality

503 in the broader consumer space.

504 Currently, virtual reality applications are being developed to provide consumers with virtual 505 teachers to automate education and training pipelines, virtual companions for the lonely, and 506 virtual therapists for those without access to mental health care. It can be imagined in these 507 applications, that the user's experience and the application's success would be influenced by 508 whether they believe there is another human on the other side of the interaction providing 509 genuine advice, friendship or support. Such beliefs could result in different degrees of value or 
510 trust placed in the utility of the training, companionship or therapy provided. Again, our

511 subjective ratings provide some tentative supporting evidence, with participants in the Human

512 group rating the task as being more pleasant, and their partner as more cooperative, than those in

513 the Computer group.

514 It is also possible that, when a virtual interaction appears and feels sufficiently real, users

515 may adopt an intentional stance, even when they know that their partner is not human. This is

516 supported by previous studies of human-robot interaction which report an association between

517 increased anthropomorphism and activation of brain regions implicated in mentalising processes

518 (Krach et al., 2008). An interesting question for future research is whether the manipulation of

519 agency beliefs has different effects depending on the type of virtual reality technology used and

520 the degree of aesthetic and behavioural realism achieved (cf. Georgescu et al., 2014). For

521 example, anthropomorphic stimuli are rated as more pleasant to look at, the more human-like

522 they appear up to the point at which they behave almost, but not exactly like real humans. It is at

523 this point that these stimuli can become aversive or subjectively unpleasant to look at - the so-

524 called "uncanny valley effect". Furthermore, the tendency to anthropomorphise might be

525 stronger for some users than others (Waytz, A., Cacioppo, J., \& Epley, N., 2010). Therefore,

526 future work is required to determine the conditions under which human agency beliefs impact on

527 the virtual reality experience and how that may vary across individuals.

528 Virtual reality is a burgeoning industry that is promising many exciting applications for

529 consumers, science and enterprise, particularly given its ability to realistically simulate social

530 interactions between single users and virtual agents. In the current study, we investigate directly

531 whether beliefs about a virtual partner's human agency can significantly influence the way in

532 which users behave and feel - and present compelling evidence that at least in some interactive 
533 contexts, it does. Software developers and researchers attempting to simulate social interactions

534 in virtual worlds need to be aware of the influence that these beliefs can have on user experience,

535 and must evaluate how this might impact (positively or negatively) on the desired goal of the

536 virtual reality application. Future research is needed to investigate how other factors such as

537 social context, degree of immersion and avatar realism impact on user experience during virtual 538 interactions.

539

540 


\section{References}

542 Caruana, N., Brock, J., \& Woolgar, A. (2015). A frontotemporoparietal network common to

543 initiating and responding to joint attention bids. Neuroimage, 108, 34-46.

$544 \quad$ http://dx.doi.org/10.1016/j.neuroimage.2014.12.041

545 Caruana, N., de Lissa, P., \& McArthur, G. (2017). Beliefs about human agency influence the

546 neural processing of gaze during joint attention. Social Neuroscience, 12 (2), 194-206.

$547 \quad$ doi: $10.1080 / 17470919.2016 .1160953$

548 Caruana, N., McArthur, G., Woolgar, A., \& Brock, J. (2017a). Simulating social interactions for 549 the experimental investigation of joint attention. Neuroscience \& Biobehavioral Reviews, 550 74, 115 - 125. doi:10.1016/j.neubiorev.2016.12.022

551 Caruana, N., McArthur, G., Woolgar, A., \& Brock, J. (2017b). Detecting communicative intent 552 in a computerised test of joint attention. PeerJ 5:e2899 https://doi.org/10.7717/peerj.2899

553 Caruana, N., Stieglitz Ham, H., Brock, J., Woolgar, A., Kloth, N., Palermo, R., \& McArthur, G. 554 (2017). Joint attention difficulties in autistic adults: An interactive eye-tracking 555 study. Autism.

556 Lee, P., \& Stewart, D. (2016). Virtual reality a billion dollar niche. Deloitte, Technology, Media 557 and Telecommunications, pp. 22-25. Retrieved from

558

559 https://www2.deloitte.com/global/en/pages/technology-media-andtelecommunications/articles/tmt-pred16-media-virtual-reality-billion-dollar560 niche.html\#full-report

561 Gallagher, H. L., Jack, A. I., Roepstorff, A., \& Frith, C. D. (2002). Imaging the intentional stance 562 in a competitive game. NeuroImage, 16, 814-821. doi:10.1006/nimg.2002.1117 
563 Georgescu, A. L., Kuzmanovic, B., Roth, D., Bente, G., \& Vogeley, K. (2014). The use of virtual

564 characters to assess and train non-verbal communication in high-functioning

565 autism. Frontiers Human Neuroscience, 807, 1-17. doi: 10.3389/fnhum.2014.00807

566 Gobel, M. S., Kim, H. S., \& Richardson, D. C. (2015). The dual function of social

567 gaze. Cognition, 136, 359-364.

568 Krach, S., Hegel, F., Wrede, B., Buschkaemper, S., Sagerer, G., \& Kircher, T. (2008). Impact of 569 differing grades of anthropomorphism and embodiment on Theory of Mind (ToM). An $570 \quad$ fMRI study. International Journal of Psychology, 43(3-4), 147-147

571 McCabe, K., Houser, D., Ryan, L., Smith, V., \& Trouard, T. (2001). A functional imaging study 572 of cooperation in two-person reciprocal exchange. Proceedings of the National Academy 573 of Sciences, 98, 11832-11835. doi: 10.1073/pnas.211415698

574 Park, S., \& Catrambone, R. (2007). Social facilitation effects of virtual humans. Human Factors: 575 The Journal of the Human Factors and Ergonomics Society, 49, 1054-1060.

576 doi: $10.1518 / 001872007 X 249910$

577 Premack, D., \& Woodruff, G. (1978). Does the chimpanzee have a theory of mind? Behavioral $578 \quad$ and Brain Sciences, 1, 515-526. https://doi.org/10.1017/S0140525

$579 \quad \mathrm{X} 00076512$

580 Pfeiffer, U. J., Schilbach, L., Timmermans, B., Kuzmanovic, B., Georgescu, A. L., Bente, G., \& 581 Vogeley, K. (2014). Why we interact: on the functional role of the striatum in the 582 subjective experience of social interaction. NeuroImage, 101, 124-137.

583 http://dx.doi.org/10.1016/j.neuroimage.2014.06.061 
584 Oberwelland, E., Schilbach, L., Barisisc, I., Krall, S. C., Vogeley, K., Fink, G. R., Herpertz-

585

586

587

588

589

590

591

592

593

594

595

596

597

598

599

600

601

602

603

604

605

606

Dahlmann, D., Konrad, K., \& Schulte-Rüther, M. (2016). Look into my eyes:

Investigating joint attention using interactive eye-tracking and $\mathrm{AMRI}$ in a developmental sample. NeuroImage, 130, 248-260.

Oldfield, R.C. (1971). The assessment and analysis of handedness: The Edinburgh Inventory. Neuropsychologia, 9, 97-113. http://dx.doi.org/10.1016/0028-3932(71)90067-4

Schilbach, L., Timmermans, B., Reddy, V., Costall, A., Bente, G., Schlicht, T., \& Vogeley, K. (2013). Toward a second-person neuroscience. Behavioral and Brain Sciences, 36, 393414. doi:10.1017/S0140525X12000660

Schilbach, L., Wilms, M., Eickhoff, S. B., Romanzetti, S., Tepest, R., Bente, G., Shah N. J., Fink, G. R., \& Vogeley, K. (2010). Minds made for sharing: Initiating joint attention recruits reward-related neurocircuitry. Journal of Cognitive Neuroscience, 22(12), 27022715. doi: 10.1162/jocn.2009.21401.

Schroeder, R. (2002). Social interaction in virtual environments: Key issues, common themes, and a framework for research. In The social life of avatars (pp. 1-18). Springer, London. SR Research. (2004). Experiment Builder (Version 1.10.165). Ontario.

Waytz, A., Cacioppo, J., \& Epley, N. (2010). Who sees human? The stability and importance of individual differences in anthropomorphism. Perspectives on Psychological Science, 5(3), 219-232.

Wykowska, A., Wiese, E., Prosser, A., Müller, H. J., \& Hamed, S. B. (2014). Beliefs about the minds of others influence how we process sensory information. PLoS ONE, 9, e94339. doi:10.1371/journal.pone.0094339 
